\title{
Model Checking. Part III
}

\author{
Kazuhisa Ishida \\ Shinshu University \\ Nagano, Japan
}

\author{
Yasunari Shidama \\ Shinshu University \\ Nagano, Japan
}

\begin{abstract}
Summary. This text includes verification of the basic algorithm in Simple On-the-fly Automatic Verification of Linear Temporal Logic (LTL). LTL formula can be transformed to Buchi automaton, and this transforming algorithm is mainly used at Simple On-the-fly Automatic Verification. In this article, we verified the transforming algorithm itself. At first, we prepared some definitions and operations for transforming. And then, we defined the Buchi automaton and verified the transforming algorithm.
\end{abstract}

MML identifier: MODELC_3, version: $\underline{7.9 .03 \quad 4.108 .1028}$

The notation and terminology used in this paper are introduced in the following articles: [5], [14], [6], [7], [1], [15], [3], [16], [2], [13], [4], [12], [10], [11], [8], and $[9]$.

\section{Definition of Basic Operations to Build an Automaton for LTL AND PROPERTIES}

For simplicity, we adopt the following rules: $k, n, m, i, j$ are elements of $\mathbb{N}$, $x, y, X$ are sets, $L, L_{1}, L_{2}$ are finite sequences, $F, H$ are LTL-formulae, $W, W_{1}$, $W_{2}$ are subsets of Subformulae $H$, and $v$ is an LTL-formula.

Let us consider $F$. Then Subformulae $F$ is a subset of $\mathrm{WFF}_{\mathrm{LTL}}$.

Let us consider $H$. The functor LTLNew $H$ yields a subset of Subformulae $H$ and is defined as follows:

(Def. 1) LTLNew $1=\left\{\begin{array}{l}\{\operatorname{Left} \operatorname{Arg}(H), \operatorname{Right} \operatorname{Arg}(H)\}, \text { if } H \text { is conjunctive, } \\ \{\operatorname{Left} \operatorname{Arg}(H)\}, \text { if } H \text { is disjunctive, } \\ \emptyset, \text { if } H \text { has next operator, } \\ \{\operatorname{Left} \operatorname{Arg}(H)\}, \text { if } H \text { has until operator, } \\ \{\operatorname{RightArg}(H)\}, \text { if } H \text { has release operator, } \\ \emptyset, \text { otherwise. }\end{array}\right.$ 
The functor LTLNew $_{2} H$ yields a subset of Subformulae $H$ and is defined as follows:

(Def. 2) LTLNew $2 H=\left\{\begin{array}{l}\emptyset, \text { if } H \text { is conjunctive, } \\ \{\operatorname{Right} \operatorname{Arg}(H)\}, \text { if } H \text { is disjunctive, } \\ \emptyset, \text { if } H \text { has } n \text { ext operator, } \\ \{\operatorname{Right} \operatorname{Arg}(H)\}, \text { if } H \text { has until operator, } \\ \{\operatorname{Left} \operatorname{Arg}(H), \operatorname{Right} \operatorname{Arg}(H)\}, \text { if } H \text { has release operator, } \\ \emptyset, \text { otherwise. }\end{array}\right.$

The functor LTLNext $H$ yielding a subset of Subformulae $H$ is defined as follows:

(Def. 3) LTLNext $H=\left\{\begin{array}{l}\{\operatorname{Arg}(H)\}, \text { if } H \text { has next operator, } \\ \{H\}, \text { if } H \text { has until operator, } \\ \{H\}, \text { if } H \text { has release operator, } \\ \emptyset, \text { otherwise. }\end{array}\right.$

Let us consider $v$. We consider LTL-nodes over $v$ as systems

$\langle$ an old-component, a new-component, a next-component 〉,

where the old-component, the new-component, and the next-component are subsets of Subformulae $v$.

Let us consider $v$, let $N$ be an LTL-node over $v$, and let us consider $H$. Let us assume that $H \in$ the new-component of $N$. The functor $\operatorname{SuccNode}_{1}(H, N)$ yielding a strict LTL-node over $v$ is defined by the conditions (Def. 4 ).

(Def. 4)(i) The old-component of $\operatorname{SuccNode}_{1}(H, N)=$ (the old-component of $N) \cup\{H\}$,

(ii) the new-component of $\operatorname{SuccNode}_{1}(H, N)=($ (the new-component of $N) \backslash\{H\}) \cup\left(\right.$ LTLNew $_{1} H \backslash$ the old-component of $\left.N\right)$, and

(iii) the next-component of $\operatorname{SuccNode}_{1}(H, N)=$ (the next-component of $N) \cup$ LTLNext $H$.

Let us consider $v$, let $N$ be an LTL-node over $v$, and let us consider $H$. Let us assume that $H \in$ the new-component of $N$ and $H$ is either disjunctive or has until operator or release operator. The functor $\operatorname{SuccNode}_{2}(H, N)$ yields a strict LTL-node over $v$ and is defined by the conditions (Def. 5).

(Def. 5)(i) The old-component of $\operatorname{SuccNode}_{2}(H, N)=$ (the old-component of $N) \cup\{H\}$,

(ii) the new-component of $\operatorname{SuccNode}_{2}(H, N)=$ ((the new-component of $N) \backslash\{H\}) \cup\left(\right.$ LTLNew $_{2} H \backslash$ the old-component of $\left.N\right)$, and

(iii) the next-component of $\operatorname{SuccNode}_{2}(H, N)=$ the next-component of $N$.

Let us consider $v$, let $N_{1}, N_{2}$ be LTL-nodes over $v$, and let us consider $H$. We say that $N_{2}$ is a successor of $N_{1}$ and $H$ if and only if the conditions (Def. 6) are satisfied.

(Def. 6)(i) $\quad H \in$ the new-component of $N_{1}$, and 
(ii) $N_{2}=\operatorname{SuccNode}_{1}\left(H, N_{1}\right)$ or $H$ is either disjunctive or has until operator or release operator and $N_{2}=\operatorname{SuccNode}_{2}\left(H, N_{1}\right)$.

Let us consider $v$ and let $N_{1}, N_{2}$ be LTL-nodes over $v$. We say that $N_{2}$ is a 1st successor of $N_{1}$ if and only if:

(Def. 7) There exists $H$ such that $H \in$ the new-component of $N_{1}$ and $N_{2}=$ $\operatorname{SuccNode}_{1}\left(H, N_{1}\right)$.

We say that $N_{2}$ is a 2 nd successor of $N_{1}$ if and only if the condition (Def. 8) is satisfied.

(Def. 8) There exists $H$ such that

(i) $\quad H \in$ the new-component of $N_{1}$,

(ii) $H$ is either disjunctive or has until operator or release operator, and

(iii) $\quad N_{2}=\operatorname{SuccNode}_{2}\left(H, N_{1}\right)$.

Let us consider $v$ and let $N_{1}, N_{2}$ be LTL-nodes over $v$. We say that $N_{2}$ is a successor of $N_{1}$ if and only if:

(Def. 9) $\quad N_{2}$ is a 1 st successor of $N_{1}$ or a 2 nd successor of $N_{1}$.

Let us consider $v$ and let $N$ be an LTL-node over $v$. We say that $N$ is failure if and only if:

(Def. 10) There exist $H, F$ such that $H$ is atomic and $F=\neg H$ and $H \in$ the old-component of $N$ and $F \in$ the old-component of $N$.

Let us consider $v$ and let $N$ be an LTL-node over $v$. We say that $N$ is elementary if and only if:

(Def. 11) The new-component of $N=\emptyset$.

Let us consider $v$ and let $N$ be an LTL-node over $v$. We say that $N$ is final if and only if:

(Def. 12) $\quad N$ is elementary and the next-component of $N=\emptyset$.

Let us consider $v$. The functor $\emptyset_{v}$ yielding a subset of Subformulae $v$ is defined as follows:

(Def. 13) $\emptyset_{v}=\emptyset$.

Let us consider $v$. The functor Seed $v$ yielding a subset of Subformulae $v$ is defined by:

(Def. 14) $\quad \operatorname{Seed} v=\{v\}$.

Let us consider $v$. Note that there exists an LTL-node over $v$ which is elementary and strict.

Let us consider $v$. The functor FinalNode $v$ yields an elementary strict LTLnode over $v$ and is defined by:

(Def. 15) FinalNode $v=\left\langle\emptyset_{v}, \emptyset_{v}, \emptyset_{v}\right\rangle$.

Let us consider $x, v$. The functor $\operatorname{CastNode}(x, v)$ yields a strict LTL-node over $v$ and is defined by: 
(Def. 16) $\operatorname{CastNode}(x, v)=\left\{\begin{array}{l}x, \text { if } x \text { is a strict LTL-node over } v \\ \left\langle\emptyset_{v}, \emptyset_{v}, \emptyset_{v}\right\rangle, \text { otherwise. }\end{array}\right.$

Let us consider $v$. The functor init $v$ yields an elementary strict LTL-node over $v$ and is defined by:

(Def. 17) init $v=\left\langle\emptyset_{v}, \emptyset_{v}\right.$, Seed $\left.v\right\rangle$.

Let us consider $v$ and let $N$ be an LTL-node over $v$. The functor $\mathcal{X} N$ yields a strict LTL-node over $v$ and is defined as follows:

(Def. 18) $\mathcal{X} N=\left\langle\emptyset_{v}\right.$, the next-component of $\left.N, \emptyset_{v}\right\rangle$.

We follow the rules: $N, N_{1}, N_{2}, M$ are strict LTL-nodes over $v$ and $w$ is an element of the infinite sequences of AtomicFamily.

Let us consider $v, L$. We say that $L$ is a successor sequence for $v$ if and only if:

(Def. 19) For every $k$ such that $1 \leq k<\operatorname{len} L$ there exist $N, M$ such that $N=L(k)$ and $M=L(k+1)$ and $M$ is a successor of $N$.

Let us consider $v, N_{1}, N_{2}$. We say that $N_{2}$ is next to $N_{1}$ if and only if the conditions (Def. 20) are satisfied.

(Def. 20)(i) $\quad N_{1}$ is elementary,

(ii) $\quad N_{2}$ is elementary, and

(iii) there exists $L$ such that $1 \leq$ len $L$ and $L$ is a successor sequence for $v$ and $L(1)=\mathcal{X} N_{1}$ and $L($ len $L)=N_{2}$.

Let us consider $v$ and let $W$ be a subset of Subformulae $v$. The functor Cast $_{\text {LTL }} W$ yielding a subset of $\mathrm{WFF}_{\mathrm{LTL}}$ is defined by:

(Def. 21) Cast $_{\mathrm{LTL}} W=W$.

Let us consider $v, N$. The functor $\cdot N$ yields a subset of $\mathrm{WFF}_{\mathrm{LTL}}$ and is defined by:

(Def. 22) $\cdot N=$ (the old-component of $N) \cup$ (the new-component of $N) \cup$ $\mathcal{X}$ Cast $_{\text {LTL }}$ (the next-component of $N$ ).

We now state three propositions:

(1) Suppose $H \in$ the new-component of $N$ and $H$ is either atomic, or negative, or conjunctive, or has next operator. Then $w=\cdot N$ if and only if $w \models \cdot \operatorname{SuccNode}_{1}(H, N)$.

(2) Suppose $H \in$ the new-component of $N$ and $H$ is either disjunctive or has until operator or release operator. Then $w=\cdot N$ if and only if one of the following conditions is satisfied:

(i) $\quad w=\cdot \operatorname{SuccNode}_{1}(H, N)$, or

(ii) $\quad w=\cdot \operatorname{SuccNode}_{2}(H, N)$.

(3) There exists $L$ such that Subformulae $H=\operatorname{rng} L$.

Let us consider $H$. Observe that Subformulae $H$ is finite. 
Let us consider $H, W, L, x$. The length of $L$ wrt $W$ and $x$ yields a natural number and is defined as follows:

(Def. 23) The length of $L$ wrt $W$ and $x=\left\{\begin{array}{l}\text { len } \operatorname{Cast}_{\mathrm{LTL}} L(x), \text { if } L(x) \in W, \\ 0, \text { otherwise. }\end{array}\right.$

Let us consider $H, W, L$. The partial sequence of $L$ wrt $W$ yields a sequence of real numbers and is defined by the condition (Def. 24).

(Def. 24) Let given $k$. Then

(i) if $L(k) \in W$, then (the partial sequence of $L$ wrt $W)(k)=$ len Cast $_{\mathrm{LTL}} L(k)$, and

(ii) if $L(k) \notin W$, then (the partial sequence of $L$ wrt $W)(k)=0$.

Let us consider $H, W, L$. The functor $\operatorname{len}(L, W)$ yields a real number and is defined as follows:

(Def. 25) $\operatorname{len}(L, W)=\sum_{\kappa=0}^{\operatorname{len} L}($ the partial sequence of $L$ wrt $W)(\kappa)$.

We now state several propositions:

(4) $\operatorname{len}\left(L, \emptyset_{H}\right)=0$.

(5) If $F \notin W$, then $\operatorname{len}(L, W \backslash\{F\})=\operatorname{len}(L, W)$.

(6) If $\operatorname{rng} L=$ Subformulae $H$ and $L$ is one-to-one and $F \in W$, then $\operatorname{len}(L, W \backslash\{F\})=\operatorname{len}(L, W)-\operatorname{len} F$.

(7) If $\operatorname{rng} L=$ Subformulae $H$ and $L$ is one-to-one and $F \notin W$ and $W_{1}=$ $W \cup\{F\}$, then len $\left(L, W_{1}\right)=\operatorname{len}(L, W)+$ len $F$.

(8) If $\operatorname{rng} L_{1}=$ Subformulae $H$ and $L_{1}$ is one-to-one and $\operatorname{rng} L_{2}=$ Subformulae $H$ and $L_{2}$ is one-to-one, then $\operatorname{len}\left(L_{1}, W\right)=\operatorname{len}\left(L_{2}, W\right)$.

Let us consider $H, W$. The functor len $W$ yields a real number and is defined by:

(Def. 26) There exists $L$ such that $\operatorname{rng} L=$ Subformulae $H$ and $L$ is one-to-one and len $W=\operatorname{len}(L, W)$.

The following propositions are true:

(9) If $F \notin W$, then $\operatorname{len}(W \backslash\{F\})=\operatorname{len} W$.

(10) If $F \in W$, then len $(W \backslash\{F\})=\operatorname{len} W-\operatorname{len} F$.

(11) If $F \notin W$ and $W_{1}=W \cup\{F\}$, then len $W_{1}=\operatorname{len} W+\operatorname{len} F$.

(12) $\operatorname{len}(W \cup\{F\}) \leq \operatorname{len} W+\operatorname{len} F$.

(13) $\operatorname{len}\left(\emptyset_{H}\right)=0$.

(14) $\operatorname{len}(\{F\})=\operatorname{len} F$.

(15) If $W \subseteq W_{1}$, then len $W \leq$ len $W_{1}$.

(16) If len $W<1$, then $W=\emptyset_{H}$.

(17) len $W \geq 0$.

(18) $\operatorname{len}\left(W_{1} \cup W_{2}\right) \leq \operatorname{len} W_{1}+\operatorname{len} W_{2}$. 
Let us consider $v, H$. Let us assume that $H \in \operatorname{Subformulae} v$. The functor $\operatorname{LTLNew}_{1}(H, v)$ yielding a subset of Subformulae $v$ is defined by:

(Def. 27) $\operatorname{LTLNew}_{1}(H, v)=$ LTLNew $_{1} H$.

The functor $\operatorname{LTLNew}_{2}(H, v)$ yields a subset of Subformulae $v$ and is defined by:

(Def. 28) $\operatorname{LTLNew}_{2}(H, v)=$ LTLNew $_{2} H$.

The following propositions are true:

(19) If $N_{2}$ is a 1st successor of $N_{1}$, then len (the new-component of $N_{2}$ ) $\leq$ len (the new-component of $N_{1}$ ) -1 .

(20) If $N_{2}$ is a 2nd successor of $N_{1}$, then len (the new-component of $N_{2}$ ) $\leq$ len (the new-component of $N_{1}$ ) -1 .

Let us consider $v, N$. The functor len $N$ yields a natural number and is defined by:

(Def. 29) $\quad \operatorname{len} N=\lfloor$ len (the new-component of $N)\rfloor$.

The following propositions are true:

(21) If $N_{2}$ is a successor of $N_{1}$, then len $N_{2} \leq$ len $N_{1}-1$.

(22) If len $N \leq 0$, then the new-component of $N=\emptyset_{v}$.

(23) If len $N>0$, then the new-component of $N \neq \emptyset_{v}$.

(24) There exist $n, L, M$ such that $1 \leq n$ and $\operatorname{len} L=n$ and $L(1)=N$ and $L(n)=M$ and the new-component of $M=\emptyset_{v}$ and $L$ is a successor sequence for $v$.

(25) Suppose $N_{2}$ is a successor of $N_{1}$. Then

(i) the old-component of $N_{1} \subseteq$ the old-component of $N_{2}$, and

(ii) the next-component of $N_{1} \subseteq$ the next-component of $N_{2}$.

(26) If $L$ is a successor sequence for $v$ and $m \leq \operatorname{len} L$ and $L_{1}=L \uparrow \operatorname{Seg} m$, then $L_{1}$ is a successor sequence for $v$.

(27) Suppose that

(i) $L$ is a successor sequence for $v$,

(ii) $F \notin$ the old-component of $\operatorname{CastNode}(L(1), v)$,

(iii) $1<n$,

(iv) $n \leq$ len $L$, and

(v) $F \in$ the old-component of CastNode $(L(n), v)$.

Then there exists $m$ such that $1 \leq m<n$ and $F \notin$ the old-component of CastNode $(L(m), v)$ and $F \in$ the old-component of CastNode $(L(m+1), v)$.

(28) Suppose $N_{2}$ is a successor of $N_{1}$ and $F \notin$ the old-component of $N_{1}$ and $F \in$ the old-component of $N_{2}$. Then $N_{2}$ is a successor of $N_{1}$ and $F$.

(29) Suppose that

(i) $\quad L$ is a successor sequence for $v$,

(ii) $F \in$ the new-component of CastNode $(L(1), v)$,

(iii) $1<n$, 
(iv) $n \leq$ len $L$, and

(v) $F \notin$ the new-component of CastNode $(L(n), v)$.

Then there exists $m$ such that $1 \leq m<n$ and $F \in$ the new-component of CastNode $(L(m), v)$ and $F \notin$ the new-component of CastNode $(L(m+$ $1), v)$.

(30) Suppose $N_{2}$ is a successor of $N_{1}$ and $F \in$ the new-component of $N_{1}$ and $F \notin$ the new-component of $N_{2}$. Then $N_{2}$ is a successor of $N_{1}$ and $F$.

(31) Suppose $L$ is a successor sequence for $v$ and $1 \leq m \leq n \leq$ len $L$. Then

(i) the old-component of $\operatorname{CastNode}(L(m), v) \subseteq$ the old-component of CastNode $(L(n), v)$, and

(ii) the next-component of CastNode $(L(m), v) \subseteq$ the next-component of CastNode $(L(n), v)$.

(32) If $N_{2}$ is a successor of $N_{1}$ and $F$, then $F \in$ the old-component of $N_{2}$.

(33) Suppose $L$ is a successor sequence for $v$ and $1 \leq \operatorname{len} L$ and the newcomponent of CastNode $(L($ len $L), v)=\emptyset_{v}$. Then the new-component of $\operatorname{CastNode}(L(1), v) \subseteq$ the old-component of CastNode $(L(\operatorname{len} L), v)$.

(34) Suppose $L$ is a successor sequence for $v$ and $1 \leq m \leq$ len $L$ and the new-component of CastNode $(L(\operatorname{len} L), v)=\emptyset_{v}$. Then the new-component of CastNode $(L(m), v) \subseteq$ the old-component of CastNode $(L(\operatorname{len} L), v)$.

(35) If $L$ is a successor sequence for $v$ and $1 \leq k<\operatorname{len} L$, then CastNode $(L(k+$ $1), v)$ is a successor of CastNode $(L(k), v)$.

(36) If $L$ is a successor sequence for $v$ and $1 \leq k \leq$ len $L$, then len $\operatorname{CastNode}(L(k), v) \leq($ len CastNode $(L(1), v)-k)+1$.

In the sequel $s, s_{0}, s_{1}, s_{2}$ denote elementary strict LTL-nodes over $v$.

The following propositions are true:

(37) If $s_{2}$ is next to $s_{1}$, then the next-component of $s_{1} \subseteq$ the old-component of $s_{2}$.

(38) Suppose $s_{2}$ is next to $s_{1}$ and $F \in$ the old-component of $s_{2}$. Then there exist $L, m$ such that

$1 \leq$ len $L$ and $L$ is a successor sequence for $v$ and $L(1)=\mathcal{X} s_{1}$ and $L($ len $L)=s_{2}$ and $1 \leq m<$ len $L$ and CastNode $(L(m+1), v)$ is a successor of CastNode $(L(m), v)$ and $F$.

(39) Suppose $s_{2}$ is next to $s_{1}$ and $H$ has release operator and $H \in$ the old-component of $s_{2}$ and $\operatorname{Left} \operatorname{Arg}(H) \notin$ the old-component of $s_{2}$. Then $\operatorname{Right} \operatorname{Arg}(H) \in$ the old-component of $s_{2}$ and $H \in$ the next-component of $s_{2}$.

(40) Suppose $s_{2}$ is next to $s_{1}$ and $H$ has release operator and $H \in$ the nextcomponent of $s_{1}$. Then RightArg $(H) \in$ the old-component of $s_{2}$ and $H \in$ the old-component of $s_{2}$.

(41) Suppose $s_{1}$ is next to $s_{0}$ and $H \in$ the old-component of $s_{1}$. Then 
(i) if $H$ is conjunctive, then $\operatorname{Left} \operatorname{Arg}(H) \in$ the old-component of $s_{1}$ and $\operatorname{Right} \operatorname{Arg}(H) \in$ the old-component of $s_{1}$,

(ii) if $H$ is either disjunctive or has until operator, then $\operatorname{Left} \operatorname{Arg}(H) \in$ the old-component of $s_{1}$ or $\operatorname{Right} \operatorname{Arg}(H) \in$ the old-component of $s_{1}$,

(iii) if $H$ has next operator, then $\operatorname{Arg}(H) \in$ the next-component of $s_{1}$, and

(iv) if $H$ has release operator, then $\operatorname{Right} \operatorname{Arg}(H) \in$ the old-component of $s_{1}$.

(42) Suppose $s_{1}$ is next to $s_{0}$ and $s_{2}$ is next to $s_{1}$ and $H \in$ the old-component of $s_{1}$ and $H$ has until operator. Then $\operatorname{Right} \operatorname{Arg}(H) \in$ the old-component of $s_{1}$ or $\operatorname{Left} \operatorname{Arg}(H) \in$ the old-component of $s_{1}$ and $H \in$ the old-component of $s_{2}$.

Let us consider $v$. The functor $\operatorname{Nodes}_{\text {LTL }} v$ yields a non empty set and is defined as follows:

(Def. 30) $\quad x \in \operatorname{Nodes}_{\mathrm{LTL}} v$ iff there exists a strict LTL-node $N$ over $v$ such that $x=N$.

Let us consider $v$. Note that $\operatorname{Nodes}_{\text {LTL }} v$ is finite.

Let us consider $v$. The functor $\operatorname{States}_{\mathrm{LTL}} v$ yields a non empty set and is defined by:

(Def. 31) States $_{\mathrm{LTL}} v=\left\{x \in\right.$ Nodes $_{\mathrm{LTL}} v: x$ is an elementary strict LTL-node over $v\}$.

Let us consider $v$. Observe that $\operatorname{States}_{\mathrm{LTL}} v$ is finite.

The following propositions are true:

(43) init $v$ is an element of States LTL $v$.

(44) $s$ is an element of States LTL $v$.

(45) $x$ is an element of States $\operatorname{LTL}_{\mathrm{L}} v$ iff there exists $s$ such that $s=x$.

Let us consider $v$, let us consider $w$, and let $f$ be a function. We say that $f$ is a successor homomorphism from $v$ to $w$ if and only if:

(Def. 32) For every $x$ such that $x \in \operatorname{Nodes}_{\text {LTL }} v$ and CastNode $(x, v)$ is non elementary and $w \models \cdot \operatorname{CastNode}(x, v)$ holds CastNode $(f(x), v)$ is a successor of CastNode $(x, v)$ and $w=\cdot \operatorname{CastNode}(f(x), v)$.

We say that $f$ is a homomorphism of $v$ into $w$ if and only if:

(Def. 33) For every $x$ such that $x \in \operatorname{Nodes}_{\text {LTL }} v$ and CastNode $(x, v)$ is non elementary and $w \models \cdot \operatorname{CastNode}(x, v)$ holds $w \models \cdot \operatorname{CastNode}(f(x), v)$.

The following propositions are true:

(46) Let $f$ be a function from $\operatorname{Nodes}_{\mathrm{LTL}} v$ into $\operatorname{Nodes}_{\mathrm{LTL}} v$. Suppose $f$ is a successor homomorphism from $v$ to $w$. Then $f$ is a homomorphism of $v$ into $w$.

(47) Let $f$ be a function from Nodes $\operatorname{LtL}_{\text {LT }} v$ into $\operatorname{Nodes}_{\text {LTL }} v$. Suppose $f$ is a homomorphism of $v$ into $w$. Let given $x$. Suppose $x \in \operatorname{Nodes}_{\mathrm{LTL}} v$ and 
CastNode $(x, v)$ is non elementary and $w \models \cdot \operatorname{CastNode}(x, v)$. Let given $k$. If for every $i$ such that $i \leq k$ holds CastNode $\left(f^{i}(x), v\right)$ is non elementary, then $w \models$. CastNode $\left(f^{k}(x), v\right)$.

(48) Let $f$ be a function from Nodes $_{\text {LTL }} v$ into Nodes $_{\text {LTL }} v$. Suppose $f$ is a successor homomorphism from $v$ to $w$. Let given $x$. Suppose $x \in \operatorname{Nodes}_{\mathrm{LTL}} v$ and $\operatorname{CastNode}(x, v)$ is non elementary and $w \models$ - CastNode $(x, v)$. Let given $k$. Suppose that for every $i$ such that $i \leq k$ holds CastNode $\left(f^{i}(x), v\right)$ is non elementary. Then CastNode $\left(f^{k+1}(x), v\right)$ is a successor of CastNode $\left(f^{k}(x), v\right)$ and $w \models \cdot \operatorname{CastNode}\left(f^{k}(x), v\right)$.

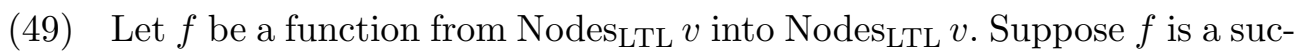
cessor homomorphism from $v$ to $w$. Let given $x$. Suppose $x \in \operatorname{Nodes}_{\mathrm{LTL}} v$ and $\operatorname{CastNode}(x, v)$ is non elementary and $w \models$. CastNode $(x, v)$. Then there exists $n$ such that for every $i$ such that $i<n$ holds CastNode $\left(f^{i}(x), v\right)$ is non elementary and CastNode $\left(f^{n}(x), v\right)$ is elementary.

(50) Let $f$ be a function from Nodes $\operatorname{stL}_{\text {L }} v$ into Nodes NTL $v$. Suppose $f$ is a homomorphism of $v$ into $w$. Let given $x$. Suppose $x \in$ $\operatorname{Nodes}_{\text {LTL }} v$ and CastNode $(x, v)$ is non elementary. Let given $k$. If CastNode $\left(f^{k}(x), v\right)$ is non elementary and $w \models \cdot \operatorname{CastNode}\left(f^{k}(x), v\right)$, then $w \models \cdot \operatorname{CastNode}\left(f^{k+1}(x), v\right)$.

(51) Let $f$ be a function from $\operatorname{Nodes}_{\text {LTL }} v$ into Nodes $L$ Lt $v$. Suppose $f$ is a successor homomorphism from $v$ to $w$. Let given $x$. Suppose $x \in \operatorname{Nodes}_{\mathrm{LTL}} v$ and $\operatorname{CastNode}(x, v)$ is non elementary and $w \models \cdot \operatorname{CastNode}(x, v)$. Then there exists $n$ such that

(i) for every $i$ such that $i<n$ holds CastNode $\left(f^{i}(x), v\right)$ is non elementary and CastNode $\left(f^{i+1}(x), v\right)$ is a successor of CastNode $\left(f^{i}(x), v\right)$,

(ii) CastNode $\left(f^{n}(x), v\right)$ is elementary, and

(iii) for every $i$ such that $i \leq n$ holds $w \models$. CastNode $\left(f^{i}(x), v\right)$.

In the sequel $q$ denotes a sequence of $\operatorname{States}_{\mathrm{LTL}} v$.

One can prove the following propositions:

(52) There exists $s$ such that $s=$ CastNode $(q(n), v)$.

(53) Suppose $H$ has until operator and $H \in$ the old-component of CastNode $(q(1), v)$ and for every $i$ holds CastNode $(q(i+1), v)$ is next to CastNode $(q(i), v)$. Suppose that for every $i$ such that $1 \leq i<n$ holds $\operatorname{Right} \operatorname{Arg}(H) \notin$ the old-component of CastNode $(q(i), v)$. Let given $i$. Suppose $1 \leq i<n$. Then $\operatorname{Left} \operatorname{Arg}(H) \in$ the old-component of CastNode $(q(i), v)$ and $H \in$ the old-component of CastNode $(q(i), v)$.

(54) Suppose $H$ has until operator and $H \in$ the old-component of CastNode $(q(1), v)$ and for every $i$ holds CastNode $(q(i+1), v)$ is next to CastNode $(q(i), v)$. Then 
(i) for every $i$ such that $i \geq 1$ holds $H \in$ the oldcomponent of CastNode $(q(i), v)$ and $\operatorname{Left} \operatorname{Arg}(H) \in$ the old-component of $\operatorname{CastNode}(q(i), v)$ and $\operatorname{Right} \operatorname{Arg}(H) \quad \notin$ the old-component of CastNode $(q(i), v)$, or

(ii) there exists $j$ such that $j \geq 1$ and $\operatorname{Right} \operatorname{Arg}(H) \in$ the old-component of CastNode $(q(j), v)$ and for every $i$ such that $1 \leq i<j$ holds $H \in$ the old-component of CastNode $(q(i), v)$ and $\operatorname{Left} \operatorname{Arg}(H) \in$ the old-component of CastNode $(q(i), v)$.

(55) $\cup\left(2_{+}^{X}\right)=X$.

(56) If $N$ is non elementary, then the new-component of $N \neq \emptyset$ and the new-component of $N \in 2_{+}^{\text {Subformulae } v}$.

Let us consider $v$. One can verify that $\bigcup\left(2_{+}^{\text {Subformulae } v}\right)$ is non empty and $2_{+}^{\text {Subformulae } v}$ is non empty.

We now state the proposition

(57) There exists a choice function of $2_{+}^{\text {Subformulae } v}$ which is a function from $2_{+}^{\text {Subformulae } v}$ into Subformulae $v$.

In the sequel $U$ denotes a choice function of $2_{+}^{\text {Subformulae } v}$.

Let us consider $v$, let us consider $U$, and let us consider $N$. Let us assume that $N$ is non elementary. The $U$-chosen formula of $N$ yielding an LTL-formula is defined as follows:

(Def. 34) The $U$-chosen formula of $N=U$ (the new-component of $N$ ).

The following proposition is true

(58) If $N$ is non elementary, then the $U$-chosen formula of $N \in$ the newcomponent of $N$.

Let us consider $w$, let us consider $v$, let us consider $U$, and let us consider $N$. The $U$-chosen successor of $N$ w.r.t. $w, v$ yields a strict LTL-node over $v$ and is defined by:

(Def. 35) The $U$-chosen successor of $N$ w.r.t. $w, v$

$$
=\left\{\begin{array}{l}
\text { SuccNode }_{1}(\text { the } U \text {-chosen formula of } N, N), \\
\text { if the } U \text {-chosen formula of } N \text { does not have until operator and } \\
w \models \cdot \operatorname{SuccNode}_{1}(\text { the } U \text {-chosen formula of } N, N) \text { or } \\
\text { the } U \text {-chosen formula of } N \text { has until operator and } \\
w \not / \operatorname{RightArg}(\text { the } U \text {-chosen formula of } N), \\
\text { SuccNode }_{2}(\text { the } U \text {-chosen formula of } N, N), \text { otherwise. }
\end{array}\right.
$$

One can prove the following propositions:

(59) Suppose $w \models \cdot N$ and $N$ is non elementary. Then

(i) $\quad w=\cdot$ (the $U$-chosen successor of $N$ w.r.t. $w, v$ ), and

(ii) the $U$-chosen successor of $N$ w.r.t. $w, v$ is a successor of $N$.

(60) Suppose $w \models \cdot N$ and $N$ is non elementary. Suppose the $U$-chosen formula of $N$ has until operator and $w=\operatorname{RightArg}($ the $U$-chosen formula of $N$ ). 
Then

(i) $\operatorname{Right} \operatorname{Arg}($ the $U$-chosen formula of $N) \in$ the new-component of the $U$-chosen successor of $N$ w.r.t. $w, v$ or RightArg(the $U$-chosen formula of $N) \in$ the old-component of $N$, and

(ii) the $U$-chosen formula of $N \in$ the old-component of the $U$-chosen successor of $N$ w.r.t. $w, v$.

(61) Suppose $w \models \cdot N$ and $N$ is non elementary. Then

(i) the old-component of $N \subseteq$ the old-component of the $U$-chosen successor of $N$ w.r.t. $w, v$, and

(ii) the next-component of $N \subseteq$ the next-component of the $U$-chosen successor of $N$ w.r.t. $w, v$.

Let us consider $w$, let us consider $v$, and let us consider $U$. The $U$-choice successor function w.r.t. $w, v$ yielding a function from $\operatorname{Nodes}_{\mathrm{LTL}} v$ into $\operatorname{Nodes}_{\mathrm{LTL}} v$ is defined by the condition (Def. 36).

(Def. 36) Let given $x$. Suppose $x \in \operatorname{Nodes}_{\text {LTL }} v$. Then (the $U$-choice successor function w.r.t. $w, v)(x)=$ the $U$-chosen successor of $\operatorname{CastNode}(x, v)$ w.r.t. $w, v$.

We now state the proposition

(62) The $U$-choice successor function w.r.t. $w, v$ is a successor homomorphism from $v$ to $w$.

\section{Negation InNer MOst LTL}

Let us consider $H$. We say that $H$ is negation-inner-most if and only if:

(Def. 37) For every LTL-formula $G$ such that $G$ is a subformula of $H$ holds if $G$ is negative, then $\operatorname{Arg}(G)$ is atomic.

Let us observe that there exists an LTL-formula which is negation-innermost.

Let us consider $H$. We say that $H$ is sub-atomic if and only if:

(Def. 38) $H$ is atomic or there exists an LTL-formula $G$ such that $G$ is atomic and $H=\neg G$.

Next we state several propositions:

(63) If $H$ is negation-inner-most and $F$ is a subformula of $H$, then $F$ is negation-inner-most.

(64) $H$ is sub-atomic iff $H$ is atomic or $H$ is negative and $\operatorname{Arg}(H)$ is atomic.

(65) Suppose $H$ is negation-inner-most. Then $H$ is either sub-atomic, or conjunctive, or disjunctive, or has next operator, or until operator, or release operator.

(66) If $H$ is negation-inner-most and has next operator, then $\operatorname{Arg}(H)$ is negation-inner-most. 
(67) Suppose that

(i) $H$ is conjunctive, or

(ii) $H$ is disjunctive, or

(iii) $H$ is negation-inner-most.

Then $\operatorname{Left} \operatorname{Arg}(H)$ is negation-inner-most and $\operatorname{Right} \operatorname{Arg}(H)$ is negationinner-most.

\section{Definition of Buchi Automaton and Verification of the Main THEOREM}

Let $W$ be a non empty set. We consider Buchi automatons over $W$ as systems

$\langle$ a carrier, a transition, an initial state, final states $\rangle$,

where the carrier is a set, the transition is a relation between the carrier $\times W$ and the carrier, the initial state is an element of $2^{\text {the carrier }}$, and the final states constitute a subset of $2^{\text {the carrier }}$.

Let $W$ be a non empty set, let $B$ be a Buchi automaton over $W$, and let $w$ be an element of the infinite sequences of $W$. We say that $w$ is accepted by $B$ if and only if the condition (Def. 39) is satisfied.

(Def. 39) There exists a sequence $r_{1}$ of the carrier of $B$ such that

(i) $\quad r_{1}(0) \in$ the initial state of $B$, and

(ii) for every natural number $i$ holds $\left\langle\left\langle r_{1}(i),(\operatorname{CastSeq}(w, W))(i)\right\rangle, r_{1}(i+\right.$ $1)\rangle \in$ the transition of $B$ and for every set $F_{1}$ such that $F_{1} \in$ the final states of $B$ holds $\left\{k \in \mathbb{N}: r_{1}(k) \in F_{1}\right\}$ is an infinite set.

For simplicity, we use the following convention: $v$ denotes a negation-innermost LTL-formula, $U$ denotes a choice function of $2_{+}^{\text {Subformulae } v}, N$ denotes a strict LTL-node over $v$, and $s, s_{1}$ denote elementary strict LTL-nodes over $v$.

Let us consider $v$ and let us consider $N$. The functor atomic LTL $N$ yields a subset of $\mathrm{WFF}_{\mathrm{LTL}}$ and is defined by:

(Def. 40) atomic LTL $_{\text {LT }}=\{x ; x$ ranges over LTL-formulae: $x$ is atomic $\wedge x \in$ the old-component of $N\}$.

The functor NegAtomic ${ }_{\text {LTL }} N$ yields a subset of $\mathrm{WFF}_{\mathrm{LTL}}$ and is defined as follows:

(Def. 41) NegAtomic $_{\text {LTL }} N=\{x ; x$ ranges over LTL-formulae: $x$ is atomic $\wedge \neg x \in$ the old-component of $N\}$.

Let us consider $v$ and let us consider $N$. The functor Label $N$ yielding a set is defined by:

(Def. 42) Label $N=\left\{x \subseteq\right.$ atomic $_{\mathrm{LTL}}$ : atomic $_{\mathrm{LTL}} N \subseteq x \wedge \operatorname{NegAtomic}_{\mathrm{LTL}} N$ misses $x\}$.

Let us consider $v$. The functor $\operatorname{Tran}_{\mathrm{LTL}} v$ yields a relation between States $_{\mathrm{LTL}} v \times$ AtomicFamily and $\operatorname{States}_{\mathrm{LTL}} v$ and is defined as follows: 
(Def. 43) $\operatorname{Tran}_{\mathrm{LTL}} v=\left\{y \in \operatorname{States}_{\mathrm{LTL}} v \times\right.$ AtomicFamily $\times \operatorname{States}_{\mathrm{LTL}} v:$ $\bigvee_{s, s_{1}, x}\left(y=\left\langle\langle s, x\rangle, s_{1}\right\rangle \wedge s_{1}\right.$ is next to $s \wedge x \in$ Label $\left.\left.s_{1}\right)\right\}$.

The functor InitS $\operatorname{StTL}_{\mathrm{LT}} v$ yielding an element of $2^{\operatorname{States}_{\mathrm{LTL}} v}$ is defined as follows:

(Def. 44) $\operatorname{InitS}_{\mathrm{LTL}} v=\{$ init $v\}$.

Let us consider $v$ and let us consider $F$. The functor $\operatorname{FinalS}_{\mathrm{LTL}}(F, v)$ yields an element of $2^{\operatorname{States}_{\text {LTL }} v}$ and is defined as follows:

(Def. 45) FinalS $\mathrm{S}_{\mathrm{LTL}}(F, v)=\left\{x \in\right.$ States $_{\mathrm{LTL}} v: F \notin$ the old-component of CastNode $(x, v) \vee \operatorname{Right} \operatorname{Arg}(F) \in$ the old-component of CastNode $(x, v)\}$.

Let us consider $v$. The functor FinalS $\mathrm{LTTL}_{\mathrm{L}} v$ yields a subset of $2^{\operatorname{States} \mathrm{LTL}} v$ and is defined by:

(Def. 46) FinalS $\mathrm{LTL}_{\mathrm{LL}} v=\left\{x \in 2^{\text {States }_{\mathrm{LTL}} v}: \bigvee_{F}(F\right.$ is a subformula of $v \wedge F$ has until operator $\left.\left.\wedge x=\operatorname{FinalS}_{\mathrm{LTL}}(F, v)\right)\right\}$.

Let us consider $v$. The functor BAutomaton $v$ yields a Buchi automaton over AtomicFamily and is defined as follows:

(Def. 47) BAutomaton $v=\left\langle\operatorname{States}_{\mathrm{LTL}} v, \operatorname{Tran}_{\mathrm{LTL}} v, \operatorname{InitS}_{\mathrm{LTL}} v\right.$, FinalS $\left.\mathrm{S}_{\mathrm{LTL}} v\right\rangle$.

The following proposition is true

(68) If $w$ is accepted by BAutomaton $v$, then $w \models v$.

Let us consider $w$, let us consider $v$, let us consider $U$, and let us consider $N$. Let us assume that $N$ is non elementary and $w \models \cdot N$. The $U$-chosen successor end number of $N$ w.r.t. $w, v$ yields an element of $\mathbb{N}$ and is defined by the conditions (Def. 48).

(Def. 48)(i) For every $i$ such that $i<$ the $U$-chosen successor end number of $N$ w.r.t. $w, v$ holds CastNode((the $U$-choice successor function w.r.t. $w$, $\left.v)^{i}(N), v\right)$ is non elementary and CastNode((the $U$-choice successor function w.r.t. $\left.w, v)^{i+1}(N), v\right)$ is a successor of CastNode((the $U$-choice successor function w.r.t. $\left.w, v)^{i}(N), v\right)$,

(ii) CastNode( (the $U$-choice successor function w.r.t.

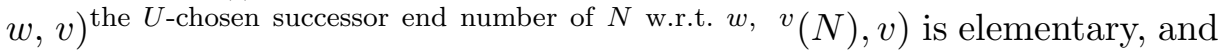

(iii) for every $i$ such that $i \leq$ the $U$-chosen successor end number of $N$ w.r.t. $w, v$ holds $w \models$. CastNode((the $U$-choice successor function w.r.t. $\left.w, v)^{i}(N), v\right)$.

Let us consider $w$, let us consider $v$, let us consider $U$, and let us consider $N$. Let us assume that $w \models \cdot \mathcal{X} N$. The $U$-chosen next node to $N$ w.r.t. $w, v$ yielding an elementary strict LTL-node over $v$ is defined by:

(Def. 49) The $U$-chosen next node to $N$ w.r.t. $w, v$

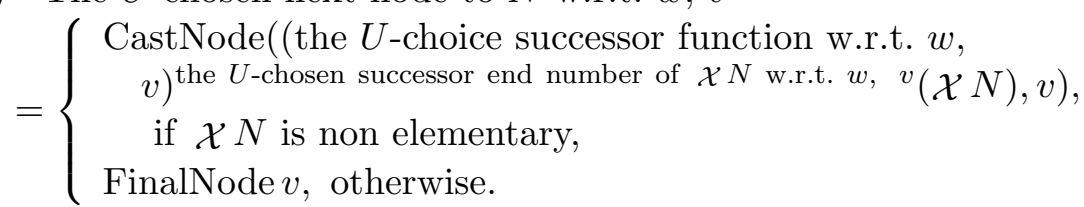

One can prove the following proposition 
(69) Suppose $w \models \cdot \mathcal{X} s$. Then the $U$-chosen next node to $s$ w.r.t. $w, v$ is next to $s$ and $w=\cdot($ the $U$-chosen next node to $s$ w.r.t. $w, v)$.

Let us consider $w$, let us consider $v$, and let us consider $U$. The $U$-chosen run w.r.t. $w, v$ yields a sequence of $\operatorname{States}_{\mathrm{LTL}} v$ and is defined by the conditions (Def. 50).

(Def. 50)(i) (The $U$-chosen run w.r.t. $w, v)(0)=$ init $v$, and

(ii) for every $n$ holds (the $U$-chosen run w.r.t. $w, v)(n+1)=$ the $U$ chosen next node to CastNode( (the $U$-chosen run w.r.t. $w, v)(n), v)$ w.r.t. $\operatorname{Shift}(w, n), v$.

The following propositions are true:

(70) If $w=\cdot N$, then $\operatorname{Shift}(w, 1) \models \cdot \mathcal{X} N$.

(71) If $w=\mathcal{X} v$, then $w=\cdot$ init $v$.

(72) $w \models v$ iff $w \models \cdot \mathcal{X}$ init $v$.

(73) Suppose $w \models v$. Let given $n$. Then

(i) CastNode((the $U$-chosen run w.r.t. $w, v)(n+1), v)$ is next to CastNode((the $U$-chosen run w.r.t. $w, v)(n), v)$, and

(ii) $\operatorname{Shift}(w, n) \models \cdot \mathcal{X}$ CastNode((the $U$-chosen run w.r.t. $w, v)(n), v)$.

(74) Suppose $w \models v$. Let given $i$. Suppose $H \in$ the old-component of CastNode( (the $U$-chosen run w.r.t. $w, v)(i+1), v)$ and $H$ has until operator and $\operatorname{Shift}(w, i)=\operatorname{Right} \operatorname{Arg}(H)$. Then $\operatorname{Right} \operatorname{Arg}(H) \in$ the old-component of CastNode( (the $U$-chosen run w.r.t. $w, v)(i+1), v)$.

(75) $\quad w$ is accepted by BAutomaton $v$ iff $w \models v$.

\section{REFERENCES}

[1] Grzegorz Bancerek. The fundamental properties of natural numbers. Formalized Mathematics, 1(1):41-46, 1990.

[2] Grzegorz Bancerek and Krzysztof Hryniewiecki. Segments of natural numbers and finite sequences. Formalized Mathematics, 1(1):107-114, 1990.

[3] Czesław Byliński. Functions and their basic properties. Formalized Mathematics, 1(1):55$65,1990$.

[4] Czesław Byliński. Functions from a set to a set. Formalized Mathematics, 1(1):153-164, 1990.

[5] Czesław Byliński. Some basic properties of sets. Formalized Mathematics, 1(1):47-53, 1990.

[6] Agata Darmochwał. Finite sets. Formalized Mathematics, 1(1):165-167, 1990.

[7] Krzysztof Hryniewiecki. Basic properties of real numbers. Formalized Mathematics, 1(1):35-40, 1990.

[8] Kazuhisa Ishida. Model checking. Part I. Formalized Mathematics, 14(4):171-186, 2006.

[9] Kazuhisa Ishida. Model checking. Part II. Formalized Mathematics, 16(3):231-245, 2008.

[10] Jarosław Kotowicz. Real sequences and basic operations on them. Formalized Mathematics, 1(2):269-272, 1990.

[11] Konrad Raczkowski and Andrzej Nȩdzusiak. Series. Formalized Mathematics, 2(4):449452, 1991.

[12] Michał J. Trybulec. Integers. Formalized Mathematics, 1(3):501-505, 1990.

[13] Wojciech A. Trybulec. Partially ordered sets. Formalized Mathematics, 1(2):313-319, 1990.

[14] Zinaida Trybulec. Properties of subsets. Formalized Mathematics, 1(1):67-71, 1990. 
[15] Edmund Woronowicz. Relations and their basic properties. Formalized Mathematics, 1(1):73-83, 1990.

[16] Edmund Woronowicz. Relations defined on sets. Formalized Mathematics, 1(1):181-186, 1990.

Received August 19, 2008 